DOI - https://doi.org/10.5965/2316796309182020036

\title{
Sistematização de diretrizes para a reabilitação virtual de idosos por meio do card sorting
}

\section{Guidelines systematization for virtual rehabilitation of the elderly through card sorting}

Carolina Bravo Pillon ${ }^{1}$ Régio Pierre da Silva ${ }^{2}$ 


\section{Resumo}

O objetivo desse artigo foi sistematizar um conjunto de diretrizes, recomendações e princípios de design para a reabilitação virtual de idosos por meio da atividade de card sorting. A metodologia incluiu quatro etapas. Na primeira etapa, foi feito um levantamento bibliográfico para a reabilitação virtual de idosos. Na segunda etapa, foi realizada a atividade de card sorting para classificar um conjunto de diretrizes conforme os grupos já definidos. $\mathrm{Na}$ terceira etapa, foi feita a análise estatística dos dados coletados. Na etapa final, as diretrizes foram reagrupadas conforme os resultados encontrados na pesquisa. A aplicação do card sorting possibilitou sistematizar um conjunto de 60 diretrizes para a reabilitação virtual de idosos em quatro grupos, sendo eles: terapia, motivação, interação e segurança.

Palavras-chave: idosos, reabilitação virtual, diretrizes, card sorting.

\section{Abstract}

The aim of this paper was to systematize a set of guidelines, recommendations, and design principles for virtual rehabilitation of the elderly through card sorting activity. The methodology included four steps. First, it was conducted a bibliographic search for virtual rehabilitation of the elderly. Second, it was done a card sorting activity in order to classify a set of guidelines according to defined groups. Third, it was done statistical analysis of the data collected. Finally, the guidelines were regrouped as the results found in the research. The card sorting application made it possible to systematize a set of 60 guidelines for the virtual rehabilitation of the elderly in four groups: therapy, motivation, interaction and safety.

Key-words: elderly, virtual rehabilitation, guidelines, card sorting.
${ }^{1}$ Mestra - UFRGS (carolinabpillon@gmail.com)

2 Doutor - UFRGS (regio@ufrgs.br)
ISSN: $2316-7963$

\section{Introdução}

As projeções demográficas indicam que em 2050 mais de $20 \%$ da população terá 60 anos ou mais, concentrando-se, especialmente, na América Latina, Ásia e China (PEDRO, 2016). Essa questão evidencia a necessidade de desenvolver produtos e serviços que possam promover o bem-estar e a qualidade de vida na velhice.

Os idosos podem, eventualmente, sofrer um declínio funcional, seja nos aspectos físico, sensorial, cognitivo e/ou psíquico. No entanto, essas condições podem ser minimizadas com o uso de recursos que podem facilitar o desempenho das atividades diárias, produtivas e de lazer (RIBEIRO; ROSÁRIO, 2016; TIRADO; BARRETO; ASSIS, 2011) Nesse contexto, a reabilitação virtual visa oferecer serviços para ajudar as pessoas a recuperar funções físicas, cognitivas e psicossociais por meio da tecnologia de realidade virtual. Os sistemas em realidade virtual permitem inserir o usuário em um ambiente virtual, oferecendo estímulos que são fundamentais para a recuperação do paciente (CAMPELO et al., 2017).

Ao desenvolver uma aplicação para a reabilitação virtual, convém adotar um conjunto de diretrizes para garantir a qualidade do artefato digital. Na visão de Camargo e Vidotti (2011), as diretrizes são recomendações ou atividades a serem realizadas ou verificadas durante o projeto. Após a coleta das informações, torna-se válido classificálas em categorias (CAMARGO; VIDOTTI, 2011). Conforme as mesmas autoras, deve-se separar o conteúdo de acordo com certas características em comum e dar um rótulo para cada categoria.

Existem algumas práticas para auxiliar nessa tarefa, entre elas pode-se citar o card sorting (CAMARGO; VIDOTTI, 2011). O arranjo de cartas, ou card sorting, é uma técnica utilizada para organizar as informações em categorias por meio de um conjunto de cartões que são distribuídos para os participantes (CYBIS; BETIOL; FAUST, 2010).

Sendo assim, o objetivo da pesquisa foi sistematizar um conjunto de diretrizes, recomendações e princípios de design para a reabilitação virtual de idosos por meio da atividade de card sorting. Para alcançar o objetivo foi delineada uma metodologia dividida em quatro etapas: revisão de literatura, coleta de dados, análise de dados e resultados. No final desse processo, foi apresentado um conjunto 60 diretrizes organizadas em quatro grupos.

Essa pesquisa pode se justificar no contexto científico/acadêmico. Pretende-se, com esse estudo, gerar um conjunto sistematizado de diretrizes para a reabilitação virtual do público sênior que, eventualmente, pode ser aplicado por designers ou outros pesquisadores para orientar o desenvolvimento de aplicações com esse propósito.

Na primeira seção, são descritos a introdução, objetivo e justificativa. Na segunda seção, é feita uma revisão de literatura a respeito do card sorting. Na terceira seção, é apresentada a metodologia da pesquisa. Na quarta seção, são mostrados os resultados alcançados. $\mathrm{Na}$ última seção, são feitas as considerações finais.

\section{Arranjo de cartas (card sorting)}

Para Camargo e Vidotti (2011), o card sorting é um método para a organização e agrupamento das informações com o uso de cartões. O objetivo é compreender as percepções do usuário em relação aos itens de conteúdo.

Durante a sessão, o pesquisador escreve cada item de informação, que, nesse caso, são as diretrizes, em fichas de papel e espalha aleatoriamente sobre a mesa. É 
solicitado que cada participante organize as fichas em grupos conforme ele julgue conveniente (CYBIS; BETIOL; FAUST, 2010). Unger e Chandler (2009) recomendam trabalhar com um número de 40 a 60 itens.

Existem duas possibilidades - o card sorting aberto e fechado (CYBIS; BETIOL; FAUST, 2010). No aberto, o participante deve fazer associações entre as cartas e nomear cada pilha de cartões. No fechado, são criados grupos previamente e os participantes devem organizar as cartas de acordo com as categorias disponíveis. Há, ainda, o remoto, o qual a sessão é feita remotamente em meio eletrônico (UNGER; CHANDLER 2009). Existem algumas ferramentas online que permitem a realização do card sorting remoto, como: OptimaSort, WebSort, xSort, SimpleCardSorting, WebCAT, Proven By Users, UsabiliTest.

A análise de dados pode ser simples, se houver uniformidade na resposta dos participantes. Para tanto, é preciso registrar a composição através de fotos ou anotações. Entretanto, muitas vezes, torna-se necessário realizar uma análise estatística para agrupar os itens de informações conforme as respostas dos participantes (CYBIS; BETIOL; FAUST, 2010). Conforme recomenda Righi et al. (2013), a análise estatística pode ser feita por meio da análise de agrupamentos (clusters), matriz de similaridade dendograma. A análise de clusters é uma técnica exploratória para identificar grupos homogêneos de itens (PESTANA; GAGEIRO, 2008).

\section{Metodologia}

Essa pesquisa pode ser classificada do tipo quantitativa. A metodologia (Figura 1) foi dividida em quatro etapas: revisão de literatura, coleta de dados, análise de dados e resultados. A pesquisa foi submetida e aprovada no Comitê de Ética em Pesquisa.

Na primeira etapa, foi feita uma revisão de literatura com o intuito de levantar diretrizes, recomendações e princípios de design para a reabilitação virtual de idosos. A busca nas bases de dados retornou 189 diretrizes. Também foram incluídas as diretrizes para o desenvolvimento de aplicações em realidade virtual propostas pelo Google Design ([S.d.]). Desse modo, foram coletadas 214 diretrizes. Foi feita uma seleção das diretrizes, resultando em 60 itens que foram utilizados na sessão de card sorting.

$\mathrm{Na}$ segunda etapa, foi realizada a sessão de card sorting com a colaboração de cinco participantes. Optou-se por realizar o card sorting fechado, no qual foram apresentados quatro grupos iniciais. Foi solicitado que os participantes classificassem o conjunto de diretrizes conforme os grupos sugeridos. Eles também deveriam indica qual a relação da diretriz com o grupo selecionado, podendo ser fraca, moderada ou forte.
Figura 1 - Metodologia da pesquisa.

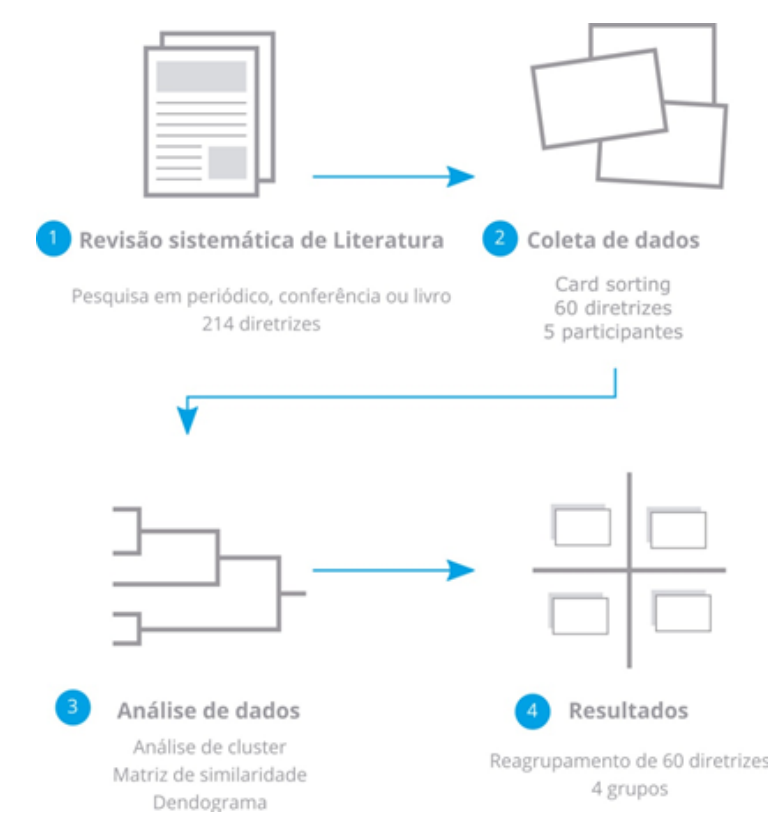

Fonte: Autoria própria (2019).

$\mathrm{Na}$ terceira etapa, foi feita a análise estatística dos dados coletados com o uso da análise de cluster, matriz de similaridade e dendograma. Os dados obtidos no card sorting foram inseridos em uma tabela no software Excel ${ }^{\circledR}$. Posteriormente, os dados foram importados no SPSS $18 \AA$. Neste, foi conduzida a análise de cluster que possibilitou gerar a matriz de similaridade e o gráfico dendograma.

Na última etapa, foi proposto o reagrupamento das diretrizes em quatro grupos: terapia, motivação, interação e segurança.

\section{Resultados}

Nessa seção, são descritos os resultados alcançados nas etapas delineadas na metodologia.

\subsection{Revisão de literatura}

Foi feita uma revisão de literatura com o objetivo de investigar quais diretrizes, recomendações ou princípios de design podem ser aplicadas para a reabilitação virtual de idosos.

A busca foi feita nas bases de dados: Proquest, Scopus, IEEE, Direct Science, Web of Science e Wiley Online Library. Os idiomas selecionados foram o Português e o Inglês. Foi utilizada a seguinte string de busca: (guidelines OR "design principles" OR recommendations) AND ("virtual rehabilitation") AND (elderly OR senior). Não foi delimitada uma data para a busca.

Os critérios de seleção foram: 1. O idioma é o Português ou o Inglês; 2. O texto está disponível em formato eletrônico; 3. Os participantes da pesquisa são idosos; 4. A pesquisa apresenta diretrizes, recomendações ou princípios de design para a reabilitação virtual.

A busca retornou 92 publicações. A inclusão e exclusão das publicações foram 
feitas com base na leitura do título, palavras-chave e resumo. As publicações que atenderam aos critérios de inclusão foram aceitas. As demais foram excluídas por não responderem à questão investigada.

Sendo assim, 20 pesquisas publicadas em periódicos, conferências ou livros foram selecionadas para a pesquisa. O Quadro 1 mostra as publicações de acordo com autor(es), ano, tipo de publicação e título.

Quadro 1 - Publicações selecionadas para a fase de coleta de diretrizes.

\begin{tabular}{|l|l|l|l|}
\hline \multicolumn{1}{|c|}{ Autor(es) } & Ano & $\begin{array}{l}\text { Periódico, Conferência ou } \\
\text { Livro }\end{array}$ & \multicolumn{1}{c|}{ Título } \\
\hline $\begin{array}{l}\text { Fernandez- } \\
\text { Cervantes et al. }\end{array}$ & 2018 & Entertainment Computing & $\begin{array}{l}\text { VirtualGym: A kinect-based } \\
\text { system for seniors exercising } \\
\text { at home }\end{array}$ \\
\hline Oña et al. & 2018 & $\begin{array}{l}\text { Computational } \\
\text { Intelligence and } \\
\text { Neuroscience }\end{array}$ & $\begin{array}{l}\text { Effectiveness of Serious } \\
\text { Games for Leap Motion on } \\
\text { the Functionality of the Upper } \\
\text { Limb in Parkinson's Disease: A } \\
\text { Feasibility Study }\end{array}$ \\
\hline $\begin{array}{l}\text { Krox, } \\
\text { Eonstantinidis Evertsen }\end{array}$ & 2017 & JMIR serious games & $\begin{array}{l}\text { User-Centered Design of } \\
\text { Serious Games for Older } \\
\text { Adults Following 3 Years of } \\
\text { Experience With Exergames } \\
\text { for Seniors: A Study Design }\end{array}$ \\
\hline Cataldi e Silva & 2017 & Design e Tecnologia & $\begin{array}{l}\text { Parâmetros para a concepção } \\
\text { e avaliação de jogos para } \\
\text { reabilitação de pacientes } \\
\text { vítimas de AVE }\end{array}$ \\
\hline Badia et al. & 2016 & $\begin{array}{l}\text { Neurorehabilitation } \\
\text { Technology }\end{array}$ & $\begin{array}{l}\text { Virtual Reality for } \\
\text { Sensorimotor Rehabilitation } \\
\text { Post Stroke: Design Principles } \\
\text { and Evidence }\end{array}$ \\
\hline Nawaz et al. & 2016 & Health Informatics Journal & $\begin{array}{l}\text { Usability and acceptability of } \\
\text { balance exergames in older } \\
\text { adults: A scoping review }\end{array}$ \\
\hline $\begin{array}{l}\text { Konstantinidis } \\
\text { et al. }\end{array}$ & 2016 & $\begin{array}{l}\text { IEEE Journal of Biomedical } \\
\text { and Health Informatics }\end{array}$ & $\begin{array}{l}\text { Design, Implementation, and } \\
\text { Wide Pilot Deployment of } \\
\text { FitForAll: An Easy to use } \\
\text { Exergaming Platform } \\
\text { Improving Physical Fitness } \\
\text { and Life Quality of Senior } \\
\text { Citizens }\end{array}$ \\
\hline & & & \\
\hline
\end{tabular}

\begin{tabular}{|c|c|c|c|}
\hline Autor(es) & Ano & $\begin{array}{l}\text { Periódico, Conferência ou } \\
\text { Livro } \\
\end{array}$ & Título \\
\hline Morán et al. & 2015 & Journal of Medical Systems & $\begin{array}{l}\text { On the Effect of Previous } \\
\text { Technological Experience on } \\
\text { the Usability of a Virtual } \\
\text { Rehabilitation Tool for the } \\
\text { Physical Activation and } \\
\text { Cognitive Stimulation of } \\
\text { Elders }\end{array}$ \\
\hline $\begin{array}{l}\text { Ramírez- } \\
\text { Fernández et } \\
\text { al. }\end{array}$ & 2014 & $\begin{array}{l}\text { ICTs for Improving Patients } \\
\text { Rehabilitation Research } \\
\text { Techniques }\end{array}$ & $\begin{array}{l}\text { Design Principles for Hapto- } \\
\text { Virtual Rehabilitation } \\
\text { Environments: Effects on } \\
\text { Effectiveness of Fine Motor } \\
\text { Hand Therapy }\end{array}$ \\
\hline Robert et al. & 2014 & $\begin{array}{l}\text { Frontiers in Aging } \\
\text { Neuroscience }\end{array}$ & $\begin{array}{l}\text { Recommendations for the } \\
\text { use of Serious Games in } \\
\text { people with Alzheimer's } \\
\text { Disease, related disorders and } \\
\text { frailty }\end{array}$ \\
\hline Nawaz et al. & 2014 & $\begin{array}{l}\text { ICTs for Improving Patients } \\
\text { Rehabilitation Research } \\
\text { Techniques }\end{array}$ & $\begin{array}{l}\text { An Exergame Concept for } \\
\text { Improving Balance in Elderly } \\
\text { People }\end{array}$ \\
\hline Uzor e Baillie & 2014 & $\begin{array}{l}\text { Proceedings of the SIGCHI } \\
\text { Conference on Human } \\
\text { Factors in Computing } \\
\text { Systems }\end{array}$ & $\begin{array}{l}\text { Investigating the Long-term } \\
\text { Use of Exergames in the } \\
\text { Home with Elderly Fallers }\end{array}$ \\
\hline Velazquez et al. & 2013 & $\begin{array}{l}\text { Proceedings of the } 2013 \\
\text { IEEE 17th International } \\
\text { Conference on Computer } \\
\text { Supported Cooperative } \\
\text { Work in Design (CSCWD) }\end{array}$ & $\begin{array}{l}\text { Design of exergames with the } \\
\text { collaborative participation of } \\
\text { older adults }\end{array}$ \\
\hline Lohse et al. & 2013 & $\begin{array}{l}\text { Journal of neurologic } \\
\text { physical therapy: JNPT }\end{array}$ & $\begin{array}{l}\text { Video games and } \\
\text { rehabilitation: using design } \\
\text { principles to enhance } \\
\text { engagement in physical } \\
\text { therapy }\end{array}$ \\
\hline $\begin{array}{l}\text { Planinc, Nake e } \\
\text { Kampel }\end{array}$ & 2013 & $\begin{array}{l}\text { The Third International } \\
\text { Conference on Ambient } \\
\text { Computing, Applications, } \\
\text { Services and Technologies }\end{array}$ & $\begin{array}{l}\text { Exergame Design Guidelines } \\
\text { for Enhancing Elderly's } \\
\text { Physical and Social Activities }\end{array}$ \\
\hline Gerling et al. & 2012 & $\begin{array}{l}\text { Proceedings of the SIGCHI } \\
\text { Conference on Human } \\
\text { Factors in Computing } \\
\text { Systems }\end{array}$ & $\begin{array}{l}\text { Full-body Motion-based } \\
\text { Game Interaction for Older } \\
\text { Adults }\end{array}$ \\
\hline Bouchard et al. & 2012 & $\begin{array}{l}\text { International Conference } \\
\text { on Serious Games } \\
\text { Development and } \\
\text { Applications }\end{array}$ & $\begin{array}{l}\text { Developing Serious Games } \\
\text { Specifically Adapted to } \\
\text { People Suffering from } \\
\text { Alzheimer }\end{array}$ \\
\hline Brox, E. et al. & 2011 & $\begin{array}{l}20115 \text { th International } \\
\text { Conference on Pervasive } \\
\text { Computing Technologies } \\
\text { for Healthcare (Pervasive } \\
\text { Health) and Workshops }\end{array}$ & $\begin{array}{l}\text { Exergames for elderly: Social } \\
\text { exergames to persuade } \\
\text { seniors to increase physical } \\
\text { activity }\end{array}$ \\
\hline
\end{tabular}

HFD, v.9, n 18, p. s6-bl, dezembro 2020 


\begin{tabular}{|l|l|l|l|}
\hline \multicolumn{1}{|c|}{ Autor(es) } & Ano & $\begin{array}{l}\text { Periódico, Conferência ou } \\
\text { Livro }\end{array}$ & \multicolumn{1}{|c|}{ Título } \\
\hline $\begin{array}{l}\text { Gerling, Schild } \\
\text { e Masuch }\end{array}$ & 2010 & $\begin{array}{l}\text { Proceedings of the 7th } \\
\text { International Conference } \\
\text { on Advances in Computer } \\
\text { Entertainment Technology }\end{array}$ & $\begin{array}{l}\text { Exergame Design for Elderly } \\
\text { Users: The Case Study of } \\
\text { SilverBalance }\end{array}$ \\
\hline $\begin{array}{l}\text { Timmermans } \\
\text { et al. }\end{array}$ & 2009 & $\begin{array}{l}\text { Journal of } \\
\text { Neuroengineering and } \\
\text { Rehabilitation }\end{array}$ & $\begin{array}{l}\text { Technology-assisted training } \\
\text { of arm-hand skills in stroke: } \\
\text { concepts on reacquisition of } \\
\text { motor control and therapist } \\
\text { guidelines for rehabilitation } \\
\text { technology design }\end{array}$ \\
\hline
\end{tabular}

Fonte: Autoria própria (2019).

No total, foram coletadas 189 diretrizes, recomendações e princípios de design As publicações abordam questões sobre o uso da reabilitação virtual para auxiliar no tratamento de algumas doenças, como Parkinson (OÑA et al., 2018), Alzheimer (ROBERT et al., 2014), Acidente Vascular Cerebral (BADIA et al., 2016). Tais estudos oferecem fundamentação teórica relevante para essa pesquisa.

Foram encontrados poucos estudos que apresentam diretrizes para o desenvolvimento de aplicações direcionadas para os óculos de realidade virtual. A maioria das pesquisas apresenta um conjunto de diretrizes para os dispositivos nãoimersivos. Sendo assim, foram incluídas 25 recomendações propostas pelo Google Design ([S.d.]). O número de dados coletadas passou a ser igual a 214.

Posteriormente, foi feita uma seleção inicial das diretrizes, o que resultou em 91 itens. As diretrizes com significado igual ou similar foram reunidas em uma mesma diretriz. 26 diretrizes foram excluídas por serem imprecisas ou visar outras tecnologias, como os sensores Leap Motion ${ }^{\circledR}$ e Kinect ${ }^{\circledR}$.

As 91 diretrizes foram analisadas pelos pesquisadores conforme a sua utilidade e compreensão. Esse critério também foi adotado por Possatti (2015). Foi atribuída uma nota de 1 a 5: quanto à utilidade, 1 - "Mínima" a 5 - "Dispensável"; e à compreensão, 1 "Não compreendi" a 5 - "Compreendi". A nota referente aos dois critérios foi somada e foram selecionadas as diretrizes com nota igual ou superior a 7.

Finalmente, foram criadas algumas categorias iniciais, incluindo: terapia, motivação, interação e segurança. Com relação à terapia (RAMÍREZ-FERNÁNDEZ et al. 2014), é importante especificar, por exemplo, quais movimentos são os mais apropriados e com que frequência eles devem ser executados. Quanto à motivação (BADIA et al., 2016), verificou-se que os elementos dos jogos podem melhorar a motivação e, se combinados com outras atividades, podem ser utilizados para engajar os usuários e, assim, alcançar os resultados desejados. No que diz respeito à interação, devemse atender às questões, como acessibilidade e usabilidade (CAMARGO; VIDOTTI, 2011), a fim de facilitar o modo como os idosos interagem com o artefato. Igualmente, é importante levar em consideração as medidas de segurança (RAMíREZ-FERNÁNDEZ et al., 2014) para evitar que o usuário sinta qualquer desconforto durante a realização da atividade. Também diz respeito à proteção dos dados do usuário.

\subsection{Coleta de dados}

Apósesse processo, 60 diretrizes foram selecionadas para o card sorting. Os cartões foram impressos e colocados aleatoriamente em um envelope que foi distribuído para os participantes. Foram realizadas 3 sessões com cinco participantes, sendo 4 alunos de pós-graduação e 1 professor do ensino superior. No início da sessão, foi solicitado que eles assinassem o TCLE. Em seguida, foram apresentados o tema da pesquisa e foram dadas as instruções para o preenchimento dos cartões. A atividade teve duração de aproximadamente 60 minutos.

O modelo do cartão utilizado no card sorting pode ser visto na Figura 2. Foi utilizado um número para facilitar a identificação das diretrizes. No espaço em branco, foi solicitado que os participantes escrevessem o grupo a qual a diretrizes poderia fazer parte. Os participantes também deveriam indicar qual a relação da diretriz com o grupo escolhido. A relação pode ser fraca (com nota igual a 1), moderada (2) ou forte (3) (HUDSON, 2005). Conforme o autor, a qualidade da relação pode ser incorporada na análise de cluster para reforçar a relação entre os itens.

Figura 2 - Modelo do cartão para a atividade de card sorting.

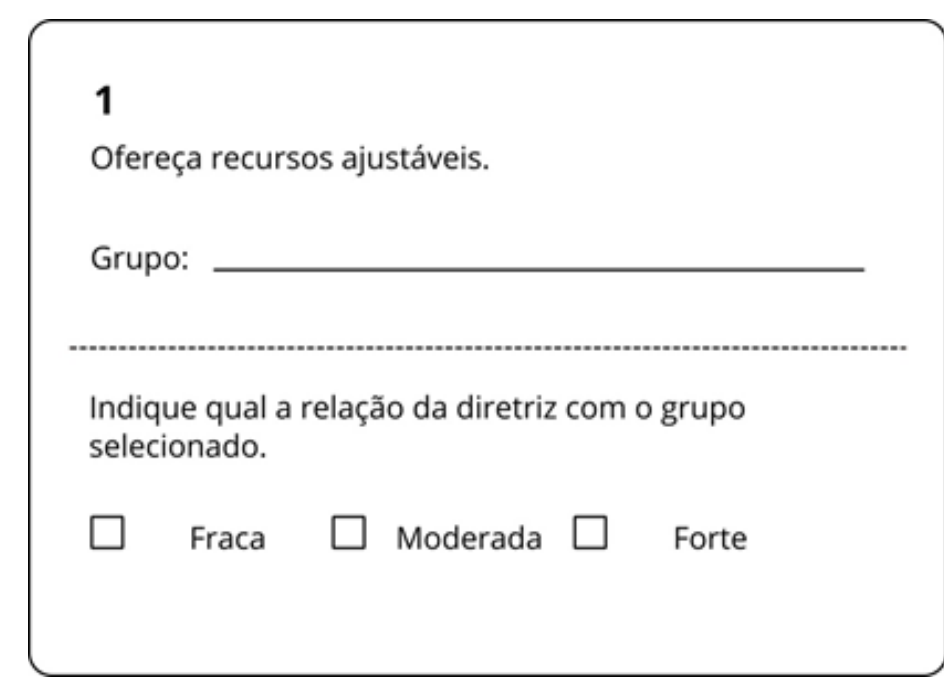

Fonte: Autoria própria (2019).

\subsection{Análise de dados}

Após a realização do card sorting, os dados foram preenchidos em uma tabela no software Excel®. As linhas representam as diretrizes e as colunas indicam os grupos. As diretrizes foram identificadas por uma letra e um número (por exemplo, D1). E os participantes foram identificados por uma letra (como, Participante A). Foi elaborada uma matriz para cada participante que, posteriormente, foram somadas e foi calculada a média das mesmas.

Essa tabela foi importada no software SPSS $18 \AA$, em que é possível gerar a análise de cluster, a matriz de similaridade e dendograma. A análise de cluster pode ser feita por meio de dois métodos: análise hierárquica e não-hierárquica. Optou-se por utilizar o método hierárquico nessa pesquisa. Para isso, deve-se acessar o menu superior do SPSS 18® e clicar em Analyse > Classify > Hierarquical Cluster (VIANA, 2017). 
As diretrizes (linhas da tabela) foram adicionadas como casos e os grupos (colunas da tabela) foram inseridos como variáveis. Em "Statistics", foram habilitadas as opções "Agglomeration schedule" e "Proximity matrix". A primeira mostra a seqüência de passos na qual o agrupamento foi realizado. A segunda gera a matriz de similaridade e representa a distância entre os pontos. Os pontos com a menor distância indicam maior semelhança e os pontos com maior distância têm maior dessemelhança. Em "Cluster Membership", foi definido o número de clusters desejados, que, nesse caso, foram quatro grupos (VIANA, 2011).

Em "Plots", foi habilitada a opção "Dendrogram" para que o software gerasse - gráfico. Trata-se de uma representação gráfica da matriz de aglomeração (Agglomeration Schedule), mostrando a seqüência como os casos foram agrupados (VIANA, 2011). A interpretação do dendograma é feita ao traçar uma linha vertical ao gráfico. A leitura do dendograma é feita da esquerda para a direita. Os pontos onde a linha intercepta o gráfico indicam o número de clusters gerados (PESTANA; GAGEIRO, 2008). O dendograma dessa pesquisa pode ser visualizado na Figura 3.

Figura 3 - Dendograma.

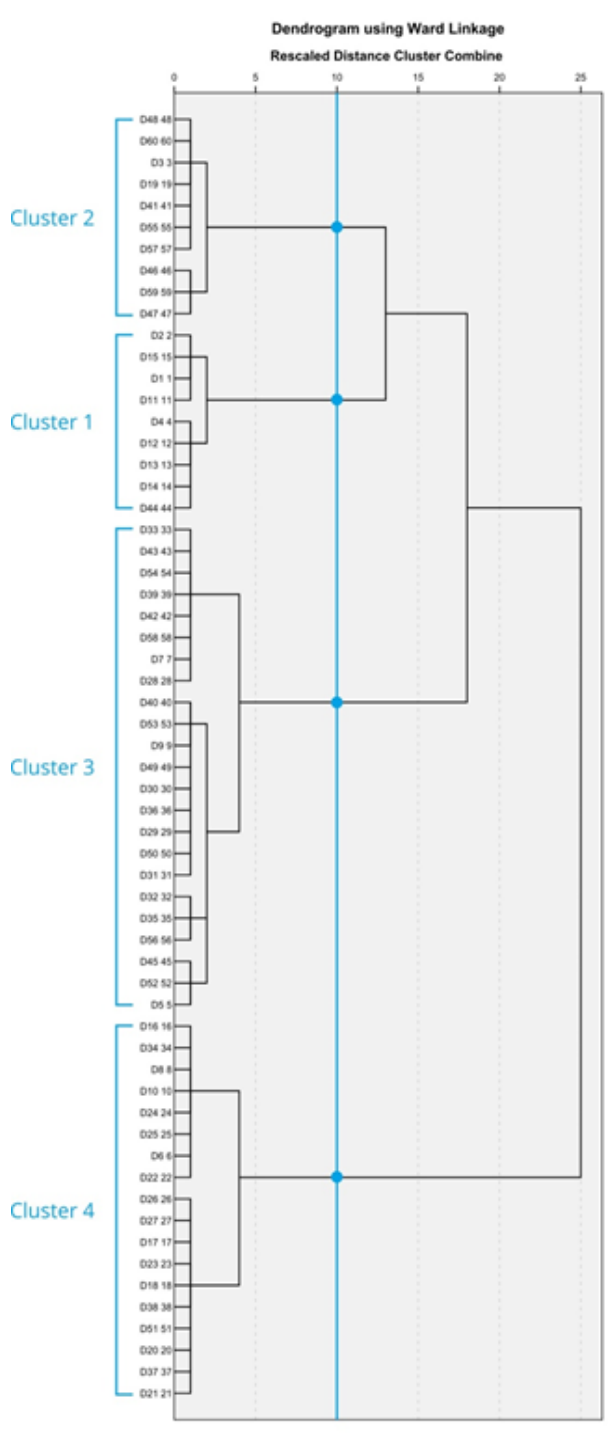

Fonte: Autoria própria (2019).
Em "Method", são definidas as técnicas de clusterização a serem utilizadas na análise. Em "Cluster Method", deve-se escolher a medida da distância que será empregada para agrupar os casos. Nessa pesquisa, foi utilizado o "Ward's Method". Também, deve-se definir a medida a ser aplicada, sendo que o tipo mais comum é a distância euclidiana ao quadrado (square euclidean distance). Em "Transform Value", é possível padronizar as variáveis, caso o pesquisador esteja trabalhando com dados diferentes, como idade, renda, escolaridade, etc. (VIANA, 2011). Nesse caso, não foi necessário fazer a padronização das variáveis, pois as mesmas estavam padronizadas conforme a escala Likert.

Em "Save", deve-se pedir para que o software crie uma variável que indicará em qual cluster os casos serão agrupados (VIANA, 2011).

\subsection{Resultados}

A partir da análise de cluster, foi gerado o dendograma, bem como a matriz de similaridade. Conforme os resultados encontrados, foi possível classificar um conjunto de 60 diretrizes em 4 grupos: terapia, segurança, interação e motivação, como pode ser visualizado no Quadro 2.

Quadro 2 - Diretrizes para os grupos: terapia, segurança, interação e movivação.

\begin{tabular}{|l|l|}
\hline ID & Diretrizes \\
\hline Cluster 1: Terapia \\
\hline D1 & Colete os dados do paciente \\
\hline D2 & Permita que os terapeutas tenham acesso aos dados \\
\hline D4 & Promova a variabilidade dos exercícios \\
\hline D11 & Inclua uma gama generalizada de movimentos que atendam a diferentes pacientes \\
\hline D12 & Incentive a repetição e a assiduidade \\
\hline D13 & Especifique as tarefas do jogo \\
\hline D14 & Promova a abstração do movimento \\
\hline D15 & Foque na qualidade do movimento sem muita distração \\
\hline D44 & Permita que o usuário controle o movimento \\
\hline Cluster 2: Segurança \\
\hline D3 & Evite o esforço excessivo \\
\hline D19 & Evite a velocidade \\
\hline D41 & Sempre mantenha o rastreamento da cabeça do usuário \\
\hline D46 & Evite mudanças bruscas na luminosidade \\
\hline D47 & Inclua pontos de referências fixos no ambiente \\
\hline D48 & Coloque o usuário em ambiente virtual estacionário \\
\hline D55 & Ofereça suporte contínuo para o jogador \\
\hline D57 & Evite mostrar dados pessoais na tela \\
\hline D59 & Evite a doença da simulação \\
\hline D60 & Utilize velocidade constante \\
\hline Cluster 3: Interação \\
\hline D5 & Favoreça a autonomia \\
\hline D7 & Use o movimento como entrada principal \\
\hline D9 & Ofereça recursos ajustáveis \\
\hline D28 & Crie uma interface clara e limpa \\
\hline
\end{tabular}




\begin{tabular}{|l|l|}
\hline D29 & Crie gráficos com cores brilhantes e bons contrastes \\
\hline D30 & Use uma interface atraente e amigável \\
\hline D31 & Use cores quentes e brilhantes com texturas simples \\
\hline D32 & Evite detalhes pequenos \\
\hline D33 & Utilize fontes grandes e claras \\
\hline D35 & Crie botões com um tamanho grande e com uma distância grande entre eles \\
\hline D36 & Use animações em vez de imagens e texto \\
\hline D39 & Ofereça feedback áudio-visual \\
\hline D40 & Forneça feedback das ações erradas \\
\hline D42 & Ofereça feedback das aços do usuário \\
\hline D43 & Coloque os controles de interface do usuário no campo de visão do usuário \\
\hline D45 & Evite objetos pequenos e com movimentos rápidos \\
\hline
\end{tabular}

\begin{tabular}{|l|l|}
\hline ID & Diretrizes \\
\hline D49 & Crie cenas simples e ajude o jogador \\
\hline D50 & $\begin{array}{l}\text { Considere usar o áudio ambiental para tornar o aplicativo mais realista e chamar a } \\
\text { atenção do usuário para várias áreas do aplicativo }\end{array}$ \\
\hline D52 & Considere incluir breves resumos de áudio para fornecer instruções aos usuários \\
\hline D53 & Forneça instruções simples e notáveis sobre como recuperar um erro \\
\hline D54 & Use diferentes tipos de entradas para fornecer assistência \\
\hline D56 & Forneça rotinas de configuração/instalação simples \\
\hline D58 & Utilize a língua nativa do usuário \\
\hline Cluster 4: Motivação \\
\hline D6 & Exiba informações para incentivar as pessoas a serem mais ativas \\
\hline D8 & Ofereça mecanismo para determinar o sucesso da terapia \\
\hline D10 & Indique com clareza quais são os resultados esperados no jogo \\
\hline D16 & Ofereça a possibilidade de ajustar a dificuldade do jogo \\
\hline D17 & Inclua elementos motivacionais no jogo \\
\hline D18 & Ofereça recompensas para as ações do jogador \\
\hline D20 & Crie uma narrativa que se aproxime das atividades da vida real dos idosos \\
\hline D21 & Mantenha o nível apropriado de desafio \\
\hline D22 & Ofereça mais tempo para que os idosos possam se envolver com o jogo \\
\hline D23 & Use um tema adequado \\
\hline D24 & Mantenha o jogador na zona de fluxo \\
\hline D25 & Ofereça objetivos claros \\
\hline D26 & Incentive a interação social \\
\hline D27 & Inclua a diversão \\
\hline D34 & Exiba informações importantes após a jogabilidade \\
\hline D37 & Comunique o progresso \\
\hline D38 & Ofereça feedback positivo \\
\hline D51 & $\begin{array}{l}\text { Forneça músicas adequadas para os idosos e a música deve se adequar ao objetivo do } \\
\text { jogo ou aos movimentos realizados }\end{array}$ \\
\hline
\end{tabular}

Fonte: Autoria própria (2019).

\section{Considerações finais}

Essa pesquisa teve como objetivo sistematizar um conjunto de diretrizes, recomendações e princípios de design para a reabilitação virtual de idosos por meio da atividade de card sorting. Para tanto, foi realizada uma pesquisa que envolveu quatro etapas: revisão de literatura, coleta de dados, análise de dados e resultados. Com isso, foi possível classificar, de forma sistemática, um conjunto de 60 diretrizes para a reabilitação virtual de idosos em quatro grupos: terapia, motivação, interação e segurança.

O grupo "Terapia" contém 9 diretrizes que estão relacionadas à fatores como coleta e análise dos dados, adaptabilidade, repetição e especificidade da tarefa. O grupo "Segurança" inclui 10 diretrizes relacionadas ao conforto e segurança, suporte e dados pessoais do usuário. O grupo "Interação" tem 23 diretrizes que dizem respeito a questões de usabilidade e acessibilidade. O grupo "Motivação" possui 18 diretrizes que visam promover a motivação do usuário durante a sessão.

Como desdobramento futuro, pretende-se aplicar o conhecimento adquirido com essa pesquisa na elaboração de um checklist, que será dividido em quatro áreas conforme os grupos mencionados. O instrumento poderá ser utilizado para orientar designers ou outros profissionais a desenvolver aplicações para a reabilitação virtual do público sênior. O checklist também poderá ser aplicado durante a sessão de teste para verificar com os usuários se o artefato atendeu às necessidades e preferências dos idosos.

\section{Agradecimento}

Os autores agradecem ao Laboratório de Virtual Design (ViD) da UFRGS. 


\section{REFERÊNCIAS}

BADIA, Sergi Bermúdez I et al. Virtual Reality for Sensorimotor Rehabilitation Post Stroke: Design Principles and Evidence. Neurorehabilitation Technology. Cham: Springer, 2016. p. 573-603.

BOUCHARD, Bruno et al. Developing Serious Games Specifically Adapted to People Suffering from Alzheimer. In: INTERNATIONAL CONFERENCE ON SERIOUS GAMES DEVELOPMENT AND APPLICATIONS, Lecture Notes in Computer Science, 2012, Berlin. Anais... Berlin: Springer, 2012. p. 243-254.

BROX, E. et al. Exergames for elderly: Social exergames to persuade seniors to increase physicalactivity. In:20115THINTERNATIONALCONFERENCEONPERVASIVECOMPUTINC TECHNOLOGIES FOR HEALTHCARE (PERVASIVEHEALTH) AND WORKSHOPS, 2011 Dublin. Anais... Dublin: IEEE, 2011. p. 546-549.

BROX, Ellen; KONSTANTINIDIS, Stathis Th; EVERTSEN, Gunn. User-Centered Design of Serious Games for Older Adults Following 3 Years of Experience With Exergames for Seniors: A Study Design. JMIR Serious Games, v. 5, n. 1, 2017.

CAMARGO, Liriane S. de A. De; VIDOTTI, Silvana A. B. G. Arquitetura da Informação: Uma Abordagem Prática para o Tratamento de Conteúdo e Interface em Ambientes Informacionais Digitais. Rio de Janeiro: LTC, 2011.

CAMPELO, A. M. et al. Virtual Rehabilitation in the elderly: Benefits, issues, and considerations. In: 2017 INTERNATIONAL CONFERENCE ON VIRTUAL REHABILITATION (ICVR), jun. 2017, Montreal. Anais... Montreal: IEEE, jun. 2017. p. 1-2.

CATALDI, Pedro Cesar Pedreira; SILVA, Tiago Barros Pontes E. Parâmetros para a concepção e avaliação de jogos para reabilitação de pacientes vítimas de AVE. Design e Tecnologia, v. 7, n. 14, p. 69, 2017

CYBIS, Walter de Abreu; BETIOL, Adriana Holtz; FAUST, Richard. Ergonomia e Usabilidade: Conhecimentos, Métodos e Aplicações. 2. ed. São Paulo: Novatec, 2010.

FERNANDEZ-CERVANTES, Victor et al. VirtualGym: A kinect-based system for seniors exercising at home. Entertainment Computing, v. 27, p. 60-72, 1 ago. 2018.

GERLING, Kathrin et al. Full-body Motion-based Game Interaction for Older Adults. CHI '12, 2012, New York. Anais... New York: ACM, 2012. p. 1873-1882.

GERLING, Kathrin Maria; SCHILD, Jonas; MASUCH, Maic. Exergame Design for Elderly Users: The Case Study of SilverBalance. ACE '10, 2010, New York, NY, USA. Anais... New York, NY, USA: ACM, 2010. p. 66-69.

GOOGLE DESIGN. A new dimension: Designing for Google Cardboard. Disponível em: <https://goo.gl/6TWsuh>. Acesso em: 24 abr. 2018.
HUDSON, William. Playing Your Cards Right: Getting the Most from Card Sorting for Navigation Design. Interactions, v. 12, n. 5, p. 56-58, set. 2005.

KONSTANTINIDIS, E. I. et al. Design, Implementation, and Wide Pilot Deployment of FitForAll: An Easy to use Exergaming Platform Improving Physical Fitness and Life Quality of Senior Citizens. IEEE Journal of Biomedical and Health Informatics, v. 20, n. 1, p. 189-200, 2016

LOHSE, Keith et al. Video games and rehabilitation: using design principles to enhance engagement in physical therapy. Journal of neurologic physical therapy: JNPT, v. 37 n. 4, p. 166-175, dez. 2013

MORÁN, Alberto L. et al. On the Effect of Previous Technological Experience on the Usability of a Virtual Rehabilitation Tool for the Physical Activation and Cognitive Stimulation of Elders. Journal of Medical Systems, v. 39, n. 9, p. 104, 2015.

NAWAZ, Ather et al. An Exergame Concept for Improving Balance in Elderly People. In INTERNATIONAL WORKSHOP ON ICTS FOR IMPROVING PATIENTS REHABILITATION RESEARCH TECHNIQUES, Communications in Computer and Information Science, 2014, Berlin. Anais... Berlin: Springer, 2014. p. 55-67.

NAWAZ, Ather et al. Usability and acceptability of balance exergames in older adults: A scoping review. Health Informatics Journal, v. 22, n. 4, p. 911-931, 2016.

OÑA, Edwin Daniel et al. Effectiveness of Serious Games for Leap Motion on the Functionality of the Upper Limb in Parkinson's Disease: A Feasibility Study. Computational Intelligence and Neuroscience, v. 2018, p. 1-17, 2018.

PEDRO, W. J. A. Processos de envelhecimento ativo e algumas dimensões sociais da ciência e tecnologia. Medicina (USP - FMRP), I Congresso de gerontecnologia. v. 49, n. 2, p. 6-7, 2016.

PESTANA, Maria Helena; GAGEIRO, João Nunes. Análise de dados para ciências sociais: a complementaridade do SPSS. 5. ed. Lisboa: Edições Sílabo, 2008.

PLANINC, Rainer; NAKE, Isabella; KAMPEL, Martin. Exergame Design Guidelines for Enhancing Elderly's Physical and Social Activities. In: THE THIRD INTERNATIONAL CONFERENCE ON AMBIENT COMPUTING, APPLICATIONS, SERVICES AND TECHNOLOGIES, 2013, Porto, Portugal. Anais... Porto, Portugal: [s.n.], 2013.

POSSATTI, Giovana Marzari. Proposta de conjunto de diretrizes editoriais para o design de livro didático digital interativo para Tablet. 2015. Dissertação (Mestrado em Design) - Universidade Federal do Rio Grande do Sul, Porto Alegre, 2015. Disponível em: <https://goo.gl/GZbwzg>. Acesso em: 8 set. 2018. 
RAMÍREZ-FERNÁNDEZ, Cristina et al. Design Principles for Hapto-Virtual Rehabilitation Environments: Effects on Effectiveness of Fine Motor Hand Therapy. In: INTERNATIONAL WORKSHOP ON ICTS FOR IMPROVING PATIENTS REHABILITATION RESEARCH TECHNIQUES, 2014, Berlin. Anais... Berlin: Springer, 2014. p. 270-284.

RIBEIRO, M. A.; ROSÁRIO, J. M. Design para todos: uma proposta de inclusão e autonomia segura. Medicina (USP - FMRP), I Congresso de gerontecnologia. v. 49, n. 2, p. 10-11, 2016.

RIGHI, Carol et al. Card Sort Analysis Best Practices. JUS - The Journal of Usability Studies, v. 8, n. 3, p. 69-89, 2013.

ROBERT, Philippe H. et al. Recommendations for the use of Serious Games in people with Alzheimer's Disease, related disorders and frailty. Frontiers in Aging Neuroscience, v. 6, 24 mar. 2014.

TIMMERMANS, Annick A. A. et al. Technology-assisted training of arm-hand skills in stroke: concepts on reacquisition of motor control and therapist guidelines for rehabilitation technology design. Journal of Neuroengineering and Rehabilitation, $v$. 6, p. 1, 2009.

TIRADO, Marcella Guimarães Assis; BARRETO, Kátia Magdala Lima; ASSIS, Luciana de Oliveira. Terapia Ocupacional em gerontologia. In: FREITAS, ELIZABETE VIANA DE; PY, LIGIA; GORZONI, MILTON LUIZ DO (Org.). Tratado de geriatria e gerontologia. 3. ed. Rio de Janeiro: Guanabara Koogan, 2011. p. 1422-1428.

UNGER, Russ; CHANDLER, Carolyn. O guia para projetar UX: A experiência do usuário (UX) para projetistas de conteúdo digital, aplicações e web sites. Rio de Janeiro: Alta Books, 2009.

UZOR, Stephen; BAILLIE, Lynne. Investigating the Long-term Use of Exergames in the Home with Elderly Fallers. CHI '14, 2014, New York. Anais... New York: ACM, 2014. p. 2813-2822.

VELAZQUEZ, A. et al. Design of exergames with the collaborative participation of older adults. In: PROCEEDINGS OF THE 2013 IEEE 17TH INTERNATIONAL CONFERENCE ON COMPUTER SUPPORTED COOPERATIVE WORK IN DESIGN (CSCWD), jun. 2013, [S.I: s.n.], jun. 2013. p. 521-526.

VIANA, Adriana Backx Noronha. Módulo 18: Análise de Cluster Tutorial SPSS Preparação dos Dados e Geração de Tabelas Método Hierárquico e Não-Hierárquico. Disponível em: <https://is.gd/guaEKZ>. Acesso em: 4 jul. 2019. , 2011 